\title{
Cinética de combustión de combustibles densificados de residuos del procesamiento de la uva isabella (Vitis labrusca L.)*
}

\author{
Andrés Felipe Rojas González** \\ Angela Viviana Ruales-Salcedo ${ }^{* *}$ \\ Francisco Javier Velasco Sarria ${ }^{* * * *}$
}

Recibido: 13/09/2016 • Aceptado: 06/12/2017

DOI: 10.22395/rium.v17n32a3

\begin{abstract}
Resumen
Este artículo presenta los resultados de la cinética de combustión por termogravimetría de briquetas preparadas con residuos de sarmientos, hollejos, escobajos y semillas, obtenidos del cultivo y procesamiento de la uva isabella (Vitis labrusca L.). Las briquetas fueron preparadas por compresión y caracterizadas por análisis próximo, análisis elemental y análisis termogravimétrico (TGA). El TGA se llevó a cabo a tres velocidades de calentamiento y en atmósfera de aire. Con los resultados del TGA se determinó la energía de activación por los métodos isoconversiones de Starink y Friedman, y el orden de reacción por el método de Avrami. Se encontró que el método Starink tiene mayor precisión que el método Friedman para el cálculo de la energía de activación. Además, los valores de orden de reacción son muy similares en los residuos y mezclas analizadas.
\end{abstract}

Palabras clave: residuos de uva; cinética de combustión; métodos iso-conversionales.

* Artículo derivado del proyecto de investigación titulado Evaluación del potencial energético y bioactivo de los residuos generados por la producción y transformación de la uva, financiado por la Universidad Nacional de Colombia con código Hermes 18921, y gracias al apoyo de la Universidad del Valle, Colombia.

** Ph. D. Profesor asociado. Departamento de Ingeniería Química. Integrante del Grupo de Investigación en Aprovechamiento de Residuos (GIAR), Universidad Nacional de Colombia, sede Manizales, Campus La Nubia, Vía al Magdalena. Teléfono: (+57+6) 8879300 ext. 55408. anfrojasgo@unal.edu.co

*** Estudiante de doctorado. M. Sc. Integrante del Grupo de Investigación en Aprovechamiento de Residuos (GIAR), Universidad Nacional de Colombia, sede Manizales, Campus La Nubia, Vía al Magdalena. Tel: $(+57+6) 887$ 9300 ext.55408. avrualess@unal.edu.co

**** Ph. D. Director Técnico, Laboratorio de Combustión Combustibles (LCC), Universidad del Valle, Ciudadela Meléndez, Cali, Colombia. francisco.velasco@correounivalle.edu.co 


\title{
Kinetics of Combustion of Densified Fuels from Residues of Isabella Grape Processing (Vitis labrusca L.)
}

\begin{abstract}
This paper presents the results of the kinetics of combustion by thermogravimetry of briquettes prepared with residues of vine shoots, skins, stalks and seeds, obtained from the cultivation and processing of Isabella grapes (Vitis labrusca L.). The briquettes were prepared by compression and characterized by near analysis, elemental analysis and thermogravimetric analysis (TGA). The TGA was carried out at three heating rates and in air atmosphere. With the results of the TGA the activation energy was determined by the Iso-conversion methods of Starink and Friedman, and the order of reaction by the Avrami method. The Starink method was found to be more accurate than the Friedman method in calculating activation energy. In addition, reaction order values are very similar for the residues and mixtures analyzed.
\end{abstract}

Keywords: grape residues; combustion kinetics; Iso-conversional methods.

\section{Cinética de combustão de combustíveis densificados de resíduos do processamento da uva Isabel (Vitis labrusca L.)}

\begin{abstract}
Resumo
Este artigo apresenta os resultados da cinética de combustão por termogravimetria de briquetes preparados com resíduos de sarmentos, bagaços, engaço e sementes, obtidos do cultivo e processamento da uva Isabel (Vitis labrusca L.). Os briquetes foram preparados por compressão e caracterizados por análise próxima, análise elementar e análise termogravimétrica (TGA). A TGA foi realizada a três velocidades de aquecimento e em atmosfera de ar. Com os resultados da TGA, determinou-se a energia de ativação pelos métodos isoconversões de Starink e Friedman, e a ordem de reação pelo método de Avrami. Constatou-se que o método Starink tem maior exatidão que o de Friedman para o cálculo da energia de ativação. Além disso, os valores de ordem de reação são muito semelhantes nos resíduos e misturas analisadas.
\end{abstract}

Palavras-chave: resíduos de uva; cinética de combustão; métodos isoconversionais. 


\section{INTRODUCCIÓN}

Los análisis termogravimétricos son realizados principalmente con dos objetivos: I) Para obtener información sobre el mecanismo de reacción, con el cual es posible modificar el curso de la reacción o predecir el comportamiento de reacciones similares, y/o II) para determinar los valores de los parámetros cinéticos de la reacción, con la finalidad de calcular velocidades de reacción por interpolación o extrapolación bajo condiciones de reacción diferentes a aquellas en las cuales se hacen los análisis [1].

Los experimentos de análisis térmico regularmente involucran reacciones heterogéneas, al menos un reactivo sólido, y aumento o disminución continuos de la temperatura (generalmente lineal). Por esto, el análisis cinético debe enfocarse en las ecuaciones de velocidad de reacciones heterogéneas bajo condiciones no isotérmicas [1]. Existen numerosas contribuciones sobre análisis cinético no isotérmico [2-3], donde la mayoría de estos métodos parten de enfoques comunes. Algunas observaciones que se deben tener presentes son: 1) problemas de medición de la temperatura de la muestra (ocasionadas por la transferencia de calor desde el horno a las regiones exteriores de la muestra y posteriormente al interior de la misma), 2) el auto-calentamiento o auto-enfriamiento de la muestra durante la reacción, y 3) la influencia de los gases producidos en la velocidad de reacción, cuando esta es altamente reversible [1].

Comúnmente se encuentra información sobre análisis termogravimétricos en procesos de pirólisis [4-8], mientras que las publicaciones sobre termogravimetría en condiciones de combustión son más limitadas [3 y 9]. Valente et al. (2015) [3] estudian la termogravimetría de residuos del proceso de elaboración de vino, en un proceso de combustión. Encontraron que la descomposición térmica de estos residuos se lleva a cabo en diferentes etapas sin formación de intermediarios estables. La variación de cada etapa está relacionada principalmente con la concentración de humedad (HM), materia volátil (MV) y carbono fijo (CF) de cada una de las muestras. Las curvas obtenidas por medio de la termogravimetría derivativa (DTG) presentan tres picos principales que corresponden al secado (evaporación del agua), a la liberación de la MV y a la degradación del CF de las muestras. En estas curvas se encontró que los escobajos presentan una velocidad de degradación térmica mayor, con un perfil de combustión más cercano al eje de las ordenadas, comparados con las curvas DTG de los hollejos y semillas. Este comportamiento indica que en procesos de combustión, este residuo presentará un tiempo de residencia en caldera menor a los hollejos y semillas [3].

El objetivo de este trabajo es establecer los parámetros cinéticos de combustión (energía de activación y orden de reacción) de combustibles densificados obtenidos de residuos del cultivo (sarmientos) y procesamiento de la uva Isabela (hollejos, escobajos y semillas) y de dos mezclas de ellos. La energía de activación se determinó empleando 
los métodos iso-conversiones de Starink y Friedman, mientras que el orden de reacción se calculó por medio del método de Avrami.

\section{MATERIALES Y MÉTODOS}

\subsection{Caracterización de las briquetas}

Los residuos de uva, escobajos (Es), hollejos (Ho), sarmientos (Sr) y semillas (Sm) se obtuvieron en una finca del Valle de Cauca (Colombia) y de una planta de producción de jugos. Estos residuos se secaron, se molieron y se compactaron en una prensa hidráulica. Los residuos compactados comúnmente se conocen como combustibles densificados o briquetas. Se prepararon cinco tipos de residuos compactos: 1) briquetas de sarmientos (BSr), 2) briquetas de hollejos (BHo), 3) briquetas de escobajos (BEs), 4) briquetas de la mezcla de residuos teniendo en cuenta la proporción en masa que se produce de $\mathrm{Sr}(32,7 \%)$ en una hectárea de cultivo y la proporción de Es $(18,7 \%)$, Ho (21,5\%) y Sm (27,1\%) que se generan en la producción de jugo de uva (BM1), y 5) briquetas de la mezcla en iguales proporciones en masa de los cuatro residuos (BM2). No se prepararon briquetas de semillas, porque estas al salir del molde de la prensa se desintegraban y no conservaban la forma de la briqueta, debido a la presencia de aceite que actúa como lubricante y evita la compactación del material. Las briquetas se caracterizaron por medio del análisis próximo (HM, MV, CF y ceniza - CZ), poder calorífico (HHV), análisis elemental (Carbono, C, Hidrógeno, H, Nitrógeno, N, Azufre, $\mathrm{S}$ y Oxígeno, $\mathrm{O}$ ) y análisis termogravimétrico. Con el análisis próximo se determinó el índice de reactividad $\mathrm{MV} / \mathrm{CF}$, el cual establece que al comparar dos muestras, la más reactiva en el proceso de combustión es aquella que presente el mayor valor de este índice.

\subsection{Análisis termogravimétrico}

Esta prueba se realiza en un analizador termogravimétrico marca TA, SDT Q600, en el que se evalúa la pérdida de peso de los residuos de uva en un proceso de combustión, a tres velocidades de calentamiento. De tal manera que se registra el cambio del peso de la muestra respecto al tiempo o la temperatura. El análisis se realiza con un tamaño de muestra entre 8 y $10 \mathrm{mg}$, en atmósfera oxidante de aire a una velocidad de flujo de $100 \mathrm{ml} / \mathrm{min}$ y a velocidades de calentamiento de 10,20 y $40{ }^{\circ} \mathrm{C} / \mathrm{min}$.

\subsection{Parámetros cinéticos de la combustión}

La energía de activación y el orden de reacción, como parámetros cinéticos de combustión en estado sólido, de las briquetas se obtienen empleando los resultados del análisis termogravimétrico. La energía de activación se calcula por medio de dos métodos 
cinéticos iso-conversionales: el método Starink y el método Friedman, mientras que el orden de reacción se evalúa utilizando la metodología de Avrami. El método Starink es una aproximación obtenida por la combinación de los métodos de Flynn-Wall-Ozawa (FWO) [10] y Kissinger-Akahira-Sunose (KAS) [11], cuyas expresiones matemáticas se muestran en las ecuaciones (1) y (2), respectivamente.

$$
\begin{gathered}
\ln \beta_{i}=\left[\frac{A E}{R G(\alpha)}\right]-2,315-0,4567 \frac{E}{R T}=C_{0}-0,4567 \frac{E}{R T} \\
\ln \left(\frac{\beta_{i}}{T_{p i}^{2}}\right)=\ln \left(\frac{A_{k} R}{E_{k}}\right)-\frac{E_{k}}{R T_{p i}}=C_{k}-\frac{E_{k}}{R T_{p i}}
\end{gathered}
$$

El método de Starink argumenta que las expresiones de aproximación del método FWO y del método KAS pueden ser transformadas en la misma fórmula general, la cual es descrita como se muestra en la ecuación (3), donde $\beta$ es la velocidad de calentamiento $(\beta=d \alpha / d T)$; $T$ es la temperatura en $\mathrm{K} ; E$ es la energía de activación, y $R$ es la constantes de los gases $(8,315 \mathrm{~J} / \mathrm{mol} \mathrm{K})$ [12]. Este método se diferencia de los métodos FWO y KAS por calcular, con mayor precisión, los valores de la energía de activación [4].

$$
\ln \left(\frac{\beta}{T^{1.8}}\right)=C_{s}-1,0037 \frac{E}{R T}
$$

El método Starink se trabaja con la conversión de la biomasa, $\alpha$, calculada mediante la ecuación (4), donde $W_{0}, W_{t}$ y $W_{\infty}$ son las masas de la muestra al inicio, al tiempo $t \mathrm{y}$ al final de la reacción, respectivamente, de tal manera que se fijan diferentes valores de conversión (entre 0 y 1 ) en los datos de TGA de las tres velocidades de calentamiento. Para cada valor fijo de conversión se lee la temperatura en cada una de las tres velocidades de calentamiento. Con estos datos se prepara un gráfico de $\ln \left(\beta / T^{1,8}\right)$ versus 1 / $T$, obteniéndose una línea recta, donde la pendiente corresponde al término $-1,0037 E / R T$ de la ecuación (3), del cual se calcula la energía de activación.

$$
\alpha=\frac{W_{0}-W_{t}}{W_{0}-W_{\infty}}
$$

El método Friedman se evalúa empleando la ecuación (5) [13], donde $A$ y $n$ representan el factor de frecuencia y el orden de reacción, respectivamente. Al igual que en el método de Starink, aquí se fijan diferentes valores de conversión y al graficar $\ln (\beta(d \alpha / d T))$ versus $1 / T$ se obtiene la pendiente, que corresponde a la energía de activación. 


$$
\ln \left(\beta \frac{d \alpha}{d T}\right)=\ln \left[A(1-\alpha)^{n}\right]-\frac{E}{R T}
$$

El orden de reacción se calcula por medio del método de Avrami dado por la ecuación (6). En la ecuación, $k$ es la constante de velocidad y está representada por la ecuación de Arrhenius: $k(T)=A \exp (-E / R T)$. Esta ecuación se manipula aplicando doble logaritmo y se obtiene la ecuación (7). Este método consiste en fijar una temperatura, y leer la conversión a dicha temperatura en los datos de TGA para cada velocidad de calentamiento. Posteriormente, se hace una gráfica de $\operatorname{Ln}(-\ln (1-\alpha))$ versus $\operatorname{Ln} \beta$ para cada temperatura fija, y se obtiene una línea recta, de cuya pendiente se calcula el orden de reacción, $n$.

$$
\begin{gathered}
\alpha=1-\exp \frac{-k(T)}{\beta^{n}} \\
\operatorname{Ln}(-\ln (1-\alpha))=\ln A-\frac{E}{R T}-n \ln \beta
\end{gathered}
$$

\section{RESULTADOS Y DISCUSIÓN}

\subsection{Caracterización de las briquetas}

Las briquetas se caracterizaron a través del análisis próximo, último y poder calorífico, como se presenta en la tabla 1 . El análisis próximo de las briquetas muestra que las BEs tienen la mayor concentración de CF $(22,15 \%)$, seguidas por las BM1 $(17,22 \%)$ y BM2 (17,52\%). El mayor porcentaje de MV se presenta en las BHo $(84,98 \%)$ y BSr $(81,82 \%)$, mientras que la mayor cantidad de cenizas se encuentran en las BEs $(6,21 \%)$. El porcentaje de humedad es similar en todas las briquetas, donde el rango varía entre 5,72 y $6,86 \%$, valores que corresponden a las BHo y BM2, respectivamente. Respecto a índice de reactividad, las $\mathrm{BHo}$ son las más reactivas $(\mathrm{MV} / \mathrm{CF}=6,90)$, lo cual es coherente con la mayor relación atómica $\mathrm{H} / \mathrm{C}$ de este briqueta, que indica un mayor contenido de estructuras moleculares con alto contenido del hidrógeno, mientras que las briquetas menos reactivas son las $\mathrm{BEs}(\mathrm{MV} / \mathrm{CF}=3,23)$. Estos tipos de cuantificaciones de la reactividad son estimaciones iniciales que deben ser corroboradas con valores de la energía de activación como medida cinética de la reactividad. 
Tabla 1. Análisis próximo, último y poder calorífico de las briquetas.

\begin{tabular}{|c|c|c|c|c|c|}
\hline & \multicolumn{5}{|c|}{ Residuo } \\
\hline & $\mathrm{BSr}$ & BEs & BHo & BM1 & BM2 \\
\hline \multicolumn{6}{|c|}{ Análisis próximo } \\
\hline$\% \mathrm{HM}$ & 6,50 & 6,30 & 5,72 & 6,19 & 6,82 \\
\hline$\% \mathrm{MV}^{\mathrm{a}}$ & 81,82 & 71,65 & 84,98 & 79,00 & 78,58 \\
\hline$\% \mathrm{CZ}^{\mathrm{a}}$ & 3,34 & 6,21 & 2,70 & 3,79 & 3,91 \\
\hline$\% \mathrm{CF}^{\mathrm{a}}$ & 14,85 & 22,15 & 12,32 & 17,22 & 17,52 \\
\hline $\mathrm{MV} / \mathrm{CF}$ & 5,51 & 3,23 & 6,90 & 4,59 & 4,49 \\
\hline $\mathrm{HHV}^{\mathrm{a}}(\mathrm{MJ} / \mathrm{kg})$ & 18,44 & 18,87 & 21,89 & 20,14 & 20,63 \\
\hline \multicolumn{6}{|c|}{ Análisis último } \\
\hline$\% \mathrm{C}^{\mathrm{b}}$ & 51,52 & 54,01 & 56,20 & 54,63 & 54,70 \\
\hline$\% \mathrm{H}^{\mathrm{b}}$ & 7,35 & 7,05 & 8,22 & 7,64 & 7,70 \\
\hline$\% \mathrm{~N}^{\mathrm{b}}$ & 0,77 & 1,86 & 1,58 & 1,60 & 1,63 \\
\hline$\% \mathrm{~S}^{\mathrm{b}}$ & 0,07 & 0,11 & 0,14 & 0,11 & 0,12 \\
\hline$\% \mathrm{O}^{\mathrm{b}}$ & 40,30 & 36,97 & 33,87 & 36,02 & 35,85 \\
\hline \multicolumn{6}{|c|}{ Relaciones atómicas } \\
\hline $\mathrm{H} / \mathrm{C}$ & 1,71 & 1,57 & 1,76 & 1,68 & 1,60 \\
\hline $\mathrm{O} / \mathrm{C}$ & 0,59 & 0,51 & 0,45 & 0,49 & 0.47 \\
\hline
\end{tabular}

${ }^{\mathrm{a}}$ Base seca; ${ }^{\mathrm{b}}$ base seca libre de cenizas

En los resultados del HHV, mostrados en la tabla 1, las BHo $(21,89 \mathrm{MJ} / \mathrm{kg})$ reportan el mayor contenido energético, seguidas por la BM1 (20,15 MJ/kg) y BM2 (20,63 MJ/ $\mathrm{kg}$ ). El mayor HHV en las BHo es congruente con los datos del análisis último, debido a que estas briquetas tienen el mayor porcentaje de $\mathrm{C} \mathrm{y} \mathrm{H}, \mathrm{y}$ menor contenido de $\mathrm{O}$, respecto a las otras muestras. Lo anterior significa que en su estructura se encuentra un mayor número de enlaces $\mathrm{C}-\mathrm{H}$, los cuales liberan más energía que los $\mathrm{C}-\mathrm{O}$, cuando son sometidos a combustión. La relación entre el HHV y el análisis último también se observa en las BSr y BEs, las cuales tienen menor concentración de $\mathrm{C}$ y mayor contenido de $\mathrm{O}$, respecto a las demás briquetas, indicando que el HHV es menor.

\subsection{Termogravimetría de los combustibles densificados}

Los análisis termogravimétricos de las briquetas se realizan en atmósfera oxidante a 10,20 , y $40{ }^{\circ} \mathrm{C} / \mathrm{min}$, como se presenta en los perfiles TG-DTG de la figura 1 . Esta 
figura muestra que las curvas TG tienen un comportamiento similar en todas las briquetas evaluadas, donde la biomasa se descompone en varias etapas sin formación de compuestos intermedios estables. De la misma manera, las curvas DTG de las briquetas presentan la misma tendencia, la cual consta de tres picos principales que corresponden a secado, desvolatilización y combustión carbonizado [1, 9 y 14].

A.

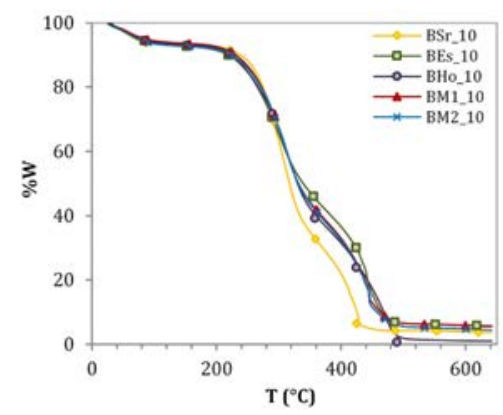

C.

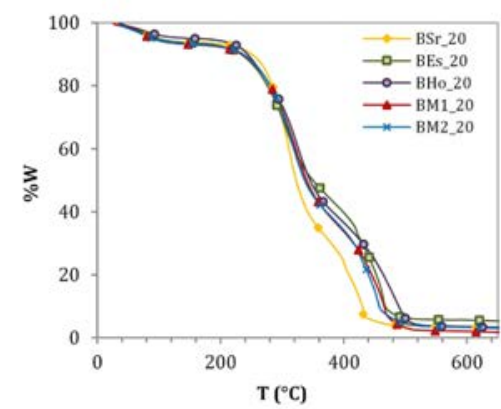

E.

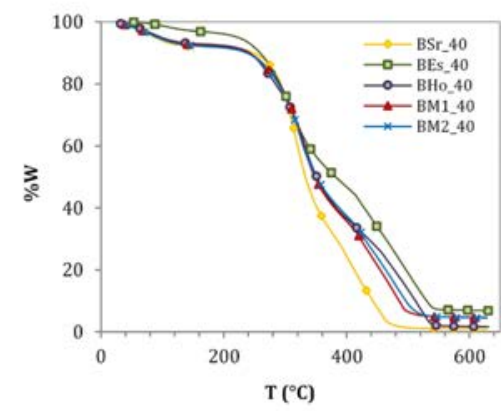

B.

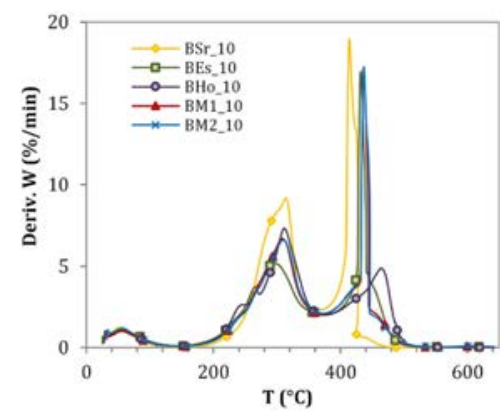

D.

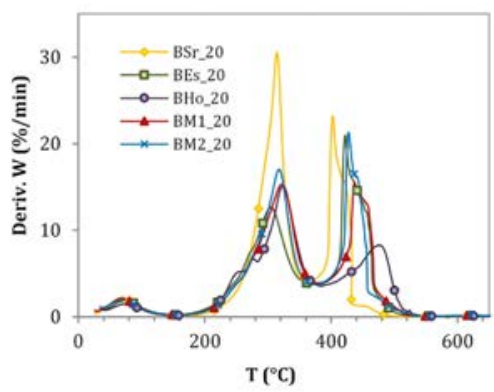

D.

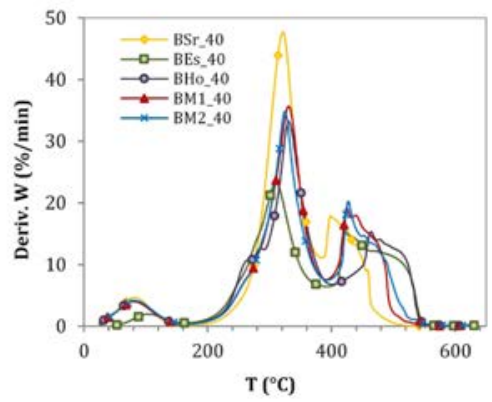

Figura 1. Curvas TG (A., C., E.) y DTG (B., D., F.) de las briquetas, realizadas a velocidades de calentamiento de 10,20 y $40^{\circ} \mathrm{C} / \mathrm{min}$. 
Con la finalidad de entender mejor el comportamiento en la degradación térmica a la combustión de las briquetas se construye la tabla 2 que reúne los parámetros: temperatura de la región de reacción $\left(\mathrm{T}_{\mathrm{rr}}\right)$, temperatura pico donde se tiene la máxima velocidad de pérdida de peso $\left(\mathrm{T}_{\mathrm{p}}\right)$, velocidad máxima de pérdida de peso $\left(\mathrm{DTG}_{\mathrm{max}}\right)$ y pérdida de masa total (PM). En esta tabla se observa que $\mathrm{T}_{\mathrm{rr}}$ aumentan al incrementar la velocidad de calentamiento. En la primera y segunda zonas, los parámetros $\mathrm{T}_{\mathrm{p}} \mathrm{y}$ DTG $_{\max }$, incrementan su valor al aumentar la velocidad de calentamiento. Sin embargo, la tercera zona no presenta una tendencia clara en estos parámetros.

Tabla 2. Parámetros de degradación térmica de las briquetas.

\begin{tabular}{|c|c|c|c|c|c|c|c|c|c|c|c|c|}
\hline \multirow[b]{2}{*}{ Muestra } & \multicolumn{4}{|c|}{$\begin{array}{c}\text { Zona I: Región de } \\
\text { evaporación }\end{array}$} & \multicolumn{4}{|c|}{$\begin{array}{c}\text { Zona II: } \\
\text { Región de reacción I }\end{array}$} & \multicolumn{4}{|c|}{$\begin{array}{c}\text { Zona III: } \\
\text { Región de reacción II }\end{array}$} \\
\hline & $\begin{array}{l}T_{r r} \\
\left({ }^{\circ} \mathrm{C}\right)\end{array}$ & $T_{p}\left({ }^{\circ} \mathrm{C}\right)$ & $\begin{array}{c}D T G_{m} \\
(\% / \\
\text { min) }\end{array}$ & $\begin{array}{l}P M \\
(\%)\end{array}$ & $T_{r r}\left({ }^{\circ} \mathrm{C}\right)$ & $T_{p}\left({ }^{\circ} \mathrm{C}\right)$ & $\begin{array}{c}D T G_{\max } \\
(\% / \\
\text { min) }\end{array}$ & $\begin{array}{l}P M \\
(\%)\end{array}$ & $T_{r r}\left({ }^{\circ} \mathrm{C}\right)$ & $T_{p}\left({ }^{\circ} \mathrm{C}\right)$ & $\begin{array}{c}D T G_{\max } \\
(\% / \\
\text { min) }\end{array}$ & $\begin{array}{l}P M \\
(\%)\end{array}$ \\
\hline \multicolumn{13}{|c|}{$\mathrm{BSr}$} \\
\hline 10 & $29-159$ & 51,3 & 1,2 & 6,8 & $159-363$ & 312,8 & 9,2 & 61,2 & $363-467$ & 413,0 & 18,5 & 27,4 \\
\hline 20 & $29-165$ & 68,8 & 2,3 & 6,2 & $165-367$ & 313,0 & 30,2 & 60,7 & $367-487$ & 402,0 & 23,2 & 29,0 \\
\hline 40 & $29-174$ & 81,3 & 4,7 & 8,0 & $174-385$ & 321,6 & 47,7 & 62,7 & $385-523$ & 398,1 & 17,9 & 28,2 \\
\hline \multicolumn{13}{|c|}{ BEs } \\
\hline 10 & $34-143$ & 53,1 & 0,2 & 7,0 & $143-371$ & 296,7 & 5,2 & 50,1 & $371-511$ & 428,7 & 16,8 & 36,2 \\
\hline 20 & $34-162$ & 78,2 & 1,1 & 6,5 & $162-376$ & 304,4 & 12,6 & 49,0 & $376-522$ & 421,9 & 20,9 & 38,6 \\
\hline 40 & $34-171$ & 106,2 & 2,0 & 3,2 & $171-397$ & 311,0 & 23,4 & 49,5 & $397-565$ & 421,3 & 15,7 & 40,1 \\
\hline \multicolumn{13}{|c|}{ ВНо } \\
\hline 10 & $32-153$ & 51,1 & 1,0 & 6,8 & $153-374$ & 311,1 & 7,3 & 56,3 & $374-519$ & 465,1 & 4,9 & 35,4 \\
\hline 20 & $32-158$ & 72,8 & 1,5 & 5,0 & $158-382$ & 323,6 & 15,1 & 55,0 & $382-537$ & 477,0 & 8,2 & 36,1 \\
\hline 40 & $32-169$ & 78,6 & 4,1 & 7,2 & $169-401$ & 331,6 & 33,1 & 56,4 & $401-558$ & 463,8 & 15,3 & 34,4 \\
\hline \multicolumn{13}{|c|}{ BM1 } \\
\hline 10 & $30-139$ & 53,2 & 1,0 & 6,3 & $139-370$ & 307,4 & 6,7 & 54,1 & $370-518$ & 435,8 & 16,9 & 33,3 \\
\hline
\end{tabular}




\begin{tabular}{|c|c|c|c|c|c|c|c|c|c|c|c|c|}
\hline \multirow[b]{2}{*}{ Muestra } & \multicolumn{4}{|c|}{$\begin{array}{c}\text { Zona I: Región de } \\
\text { evaporación }\end{array}$} & \multicolumn{4}{|c|}{$\begin{array}{c}\text { Zona II: } \\
\text { Región de reacción I }\end{array}$} & \multicolumn{4}{|c|}{$\begin{array}{c}\text { Zona III: } \\
\text { Región de reacción II }\end{array}$} \\
\hline & $\begin{array}{c}T_{r r} \\
\left({ }^{\circ} \mathrm{C}\right)\end{array}$ & $T_{p}\left({ }^{\circ} \mathrm{C}\right)$ & $\begin{array}{c}D T G_{m} \\
(\% / \\
\text { min) }\end{array}$ & $\begin{array}{l}P M \\
(\%)\end{array}$ & $T_{r r}\left({ }^{\circ} \mathrm{C}\right)$ & $T_{p}\left({ }^{\circ} \mathrm{C}\right)$ & $\begin{array}{c}D T G_{\max } \\
(\% / \\
\text { min) }\end{array}$ & $\begin{array}{l}P M \\
(\%)\end{array}$ & $T_{r r}\left({ }^{\circ} \mathrm{C}\right)$ & $T_{p}\left({ }^{\circ} \mathrm{C}\right)$ & $\begin{array}{c}D T G_{\max } \\
(\% / \\
\text { min) }\end{array}$ & $\begin{array}{l}P M \\
(\%)\end{array}$ \\
\hline 20 & $30-156$ & 65,9 & 2,1 & 6,8 & $156-381$ & 319,3 & 15,2 & 55,3 & $381-523$ & 435,3 & 15,0 & 35,3 \\
\hline 40 & $30-173$ & 78,4 & 4,0 & 7,7 & $173-393$ & 328,2 & 35,3 & 55,1 & $393-554$ & 422,4 & 18,6 & 32,3 \\
\hline \multicolumn{13}{|c|}{ BM2 } \\
\hline 10 & $33-139$ & 53,8 & 1,2 & 7,2 & $139-368$ & 309,9 & 6,5 & 53,3 & $368-518$ & 436,1 & 16,9 & 34,2 \\
\hline 20 & $33-157$ & 67,9 & 1,9 & 6,8 & $157-376$ & 314,8 & 16,8 & 54,0 & $376-533$ & 426,4 & 21,0 & 35,5 \\
\hline 40 & $33-173$ & 79,9 & 4,2 & 8,0 & $173-394$ & 323,0 & 34,5 & 53,4 & $394-554$ & 427,5 & 20,3 & 33,8 \\
\hline
\end{tabular}

$\mathrm{T}_{\mathrm{rr}}$ :Temperatura inicial y final de la región, $\mathrm{T}_{\mathrm{p}}$ : Temperatura de la máxima pérdida de peso, $\mathrm{DTG}_{\max }$ : Máxima velocidad de pérdida de peso, ML: Pérdida de peso total.

Fuente: elaboración propia

Por otra parte, el parámetro PM muestra pequeñas fluctuaciones en las diferentes velocidades de calentamiento evaluadas. Este comportamiento también es reportado por Jeguirim y Trouvé (2009) [15] quienes explican que la pérdida de masa en cualquier zona está determinada principalmente por los componentes mayoritarios de la muestra evaluada, lo cual no se ve afectado por la velocidad de calentamiento.

Si se compara los perfiles DTG a $20^{\circ} \mathrm{C} / \mathrm{min}$ de las briquetas, se puede observar que la temperatura a la que inicia la desvolatilización es similar en todas las muestras $\left(156-165{ }^{\circ} \mathrm{C}\right)$. Sin embargo, la temperatura final de la combustión del carbonizado (región de reacción II) varía de 487 a $537^{\circ} \mathrm{C}$, valores que corresponden a las $\mathrm{BSr}$ y $\mathrm{BHo}$, respectivamente. Estos resultados indican que el tiempo de residencia de las $\mathrm{BHo}$ es mayor que el resto de briquetas en el proceso de combustión; por tanto, este tipo de briqueta requiere más tiempo para entregar la energía que contiene.

\subsection{Parámetros cinéticos}

Con los resultados del análisis termogravimétrico de las briquetas se determina la energía de activación siguiendo los métodos Starink y Friedman, y el orden de reacción, por medio de la metodología de Avrami. En las figuras 2 y 3 se presentan las líneas de regresión (con coeficientes de regresión lineal cercanos a la unidad) calculadas a cada una de las briquetas, empleando los métodos Starink y Friedman, respectivamente, para valores de conversión fijos entre 0,2 y 0,8 . 

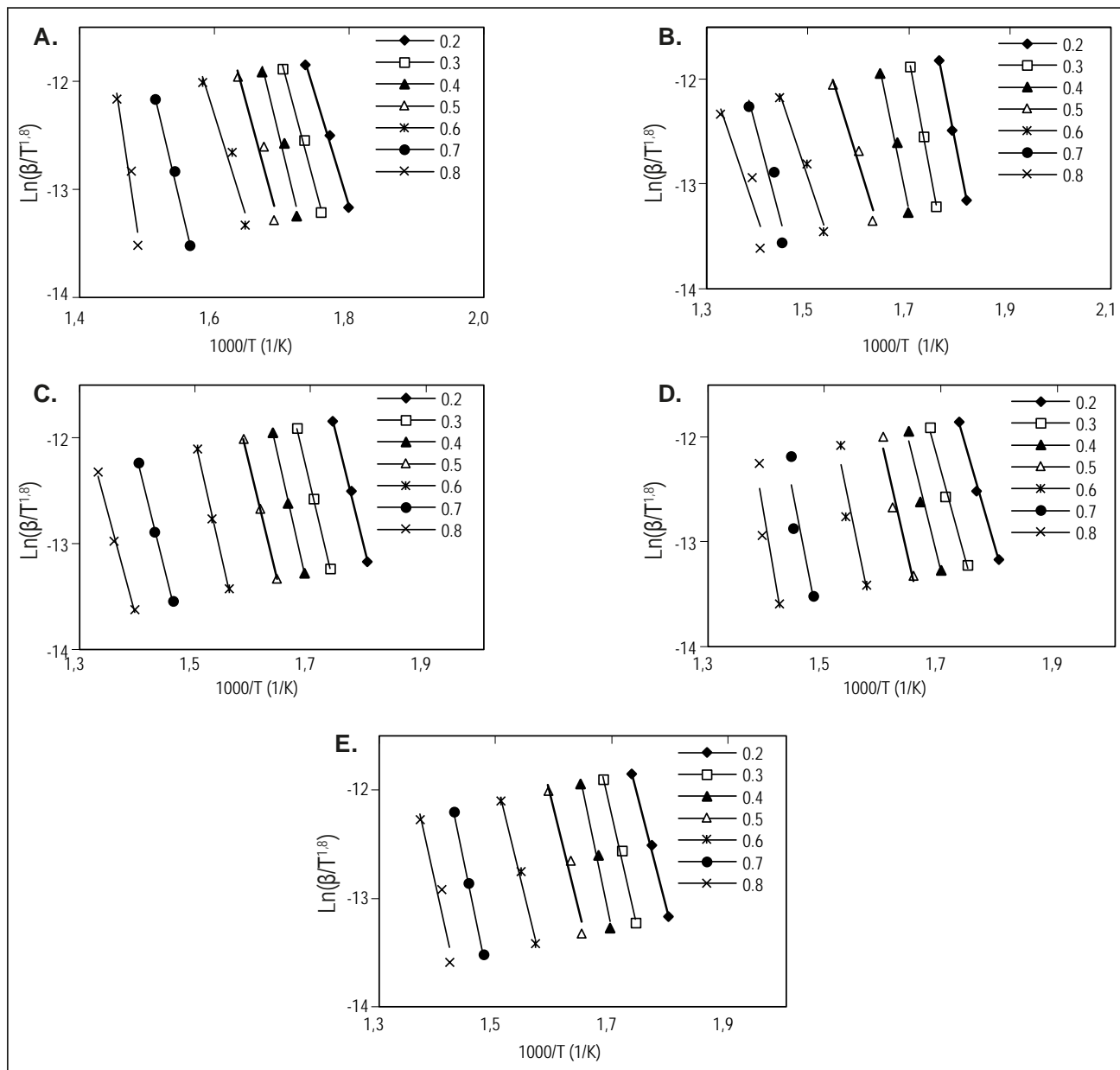

Figura 2. Regresión lineal de la energía de activación propuesta por el método Starink.

A. BSr, B. BEs, C. Bho, D. BM1, E. BM2.

Fuente: elaboración propia

A partir de las figuras 2 y 3, y las ecuaciones (3) y (5), se determina la energía de activación para cada método, y se evalúa la variación de la energía de activación con la conversión, como se muestra en las figuras 4 y 5. 


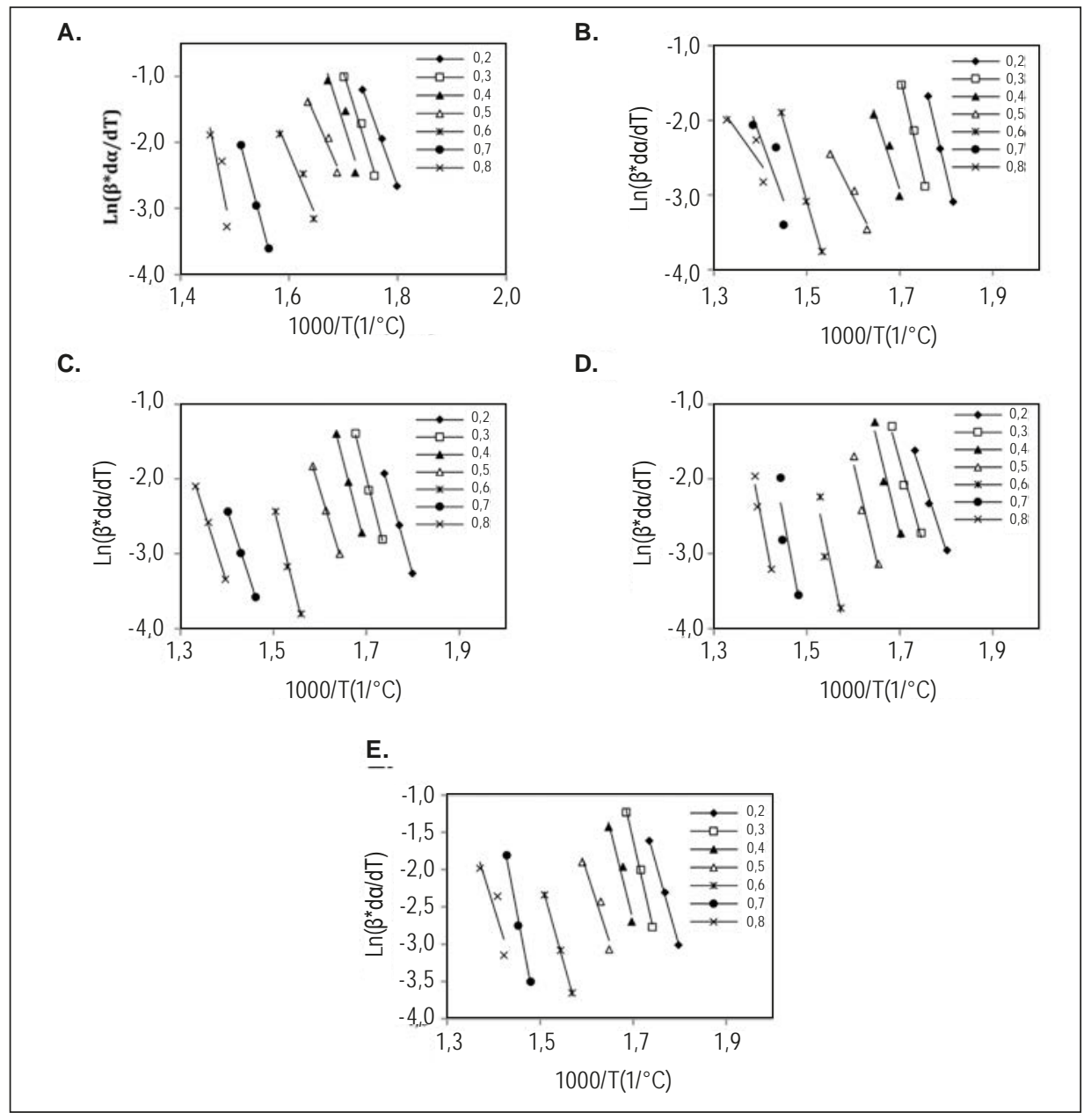

Figura 3. Regresión lineal de la energía de activación propuesta por el método Friedman. A. BSr, B. BEs, C. BHo, D. BM1, E. BM2.

Fuente: elaboración propia. 


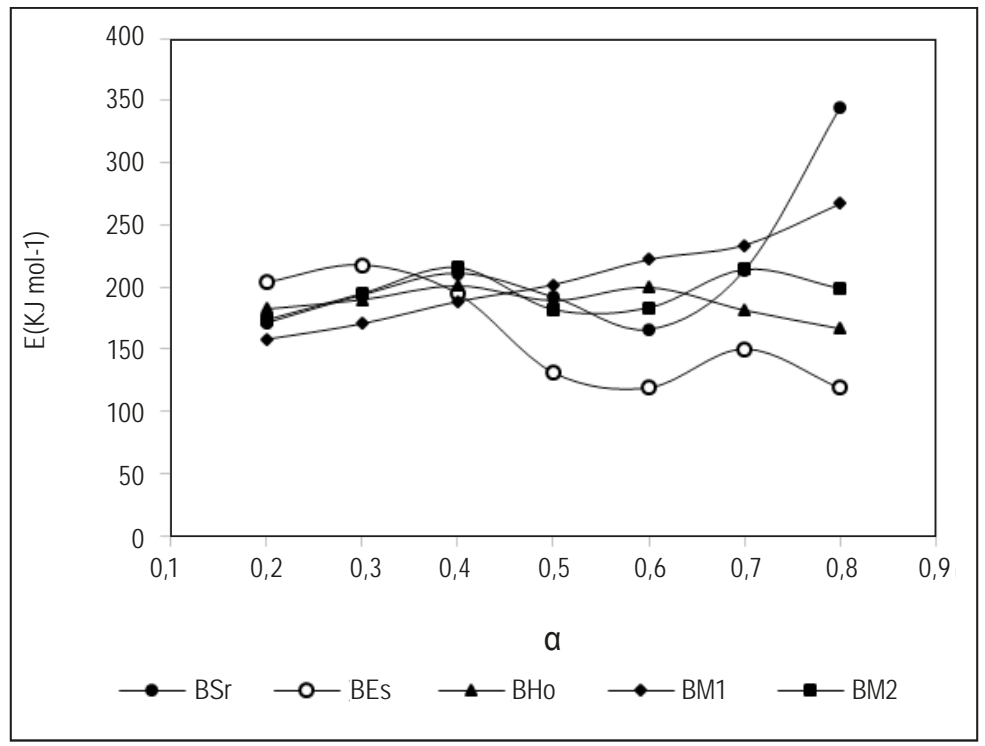

Figura 4. Energía de activación método Starink.

Fuente: elaboración propia

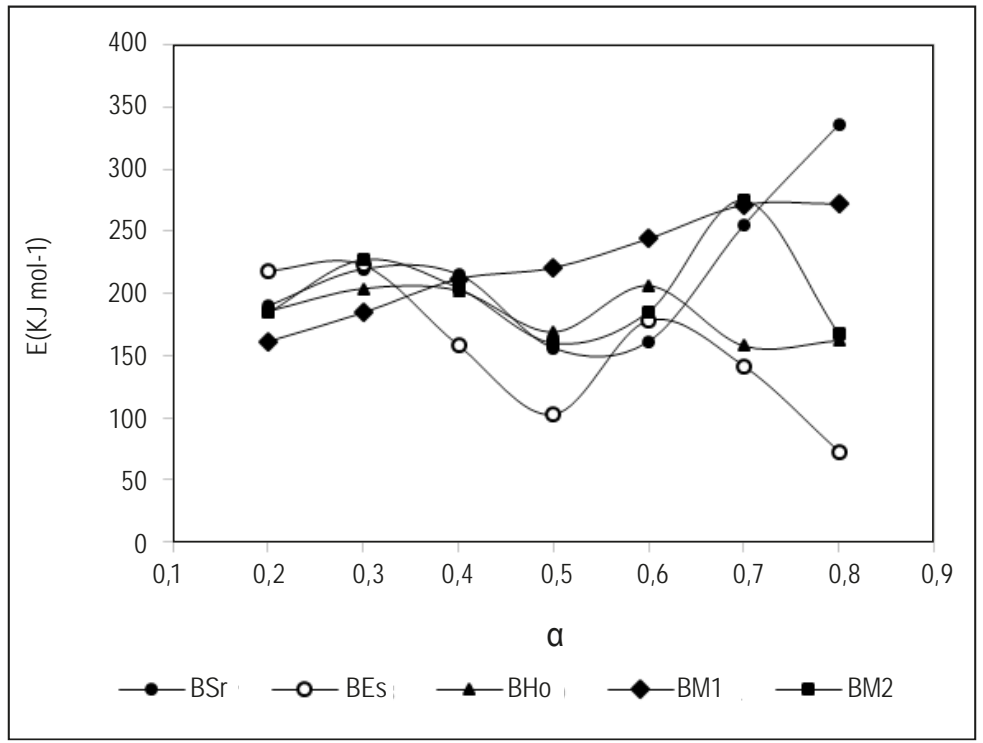

Figura 5. Energía de activación método Friedman.

Fuente: elaboración propia

En las dos figuras se observa que la energía de activación de las cinco briquetas evaluadas varía entre las diferentes conversiones, en los dos métodos. Este compor- 
tamiento se debe a que la biomasa presenta diferentes componentes que generan numerosas reacciones en paralelo y consecutivas [2 y 9]. Gai et al. (2013) [4] reportan valores de energía de activación similares a los encontrados en el presente trabajo, los cuales fueron calculados a residuos agrícolas empleando el método Starink. También se aprecia que las energías de activación de cada uno de los residuos, obtenidas por el método Starink y el método Friedman a la misma conversión, presentan pequeñas diferencias.

En la tabla 3 se presenta la energía de activación promedio determinada por los métodos Starink y Friedman, y el orden de reacción para cada una de las briquetas. En esta tabla se observa que los valores de energía de activación por los dos métodos son ligeramente cercanos, pero al comparar las desviaciones estándar de cada residuo, se encentra que son menores en el método de Starink. Esto confirma el hecho que el método Starink presenta mayor precisión en los valores de energía de activación comparado con otros métodos [4].

Tabla 3. Parámetros cinéticos de combustión de las briquetas.

\begin{tabular}{|cccc|}
\hline \multirow{2}{*}{ Briqueta } & \multicolumn{2}{c}{$E, \mathrm{~kJ} / \mathrm{mol}$} & Orden de reacción \\
\cline { 2 - 3 } & M. Starink & M. Friedman & $n$ \\
\hline BSr & $213,4 \pm 60,6$ & $219,3 \pm 62,0$ & 0,33 \\
BEs & $162,4 \pm 42,4$ & $156,3 \pm 56,0$ & 0,27 \\
BHo & $187,3 \pm 11,8$ & $184,8 \pm 20,9$ & 0,28 \\
BM1 & $206,1 \pm 37,9$ & $224,6 \pm 42,0$ & 0,27 \\
BM2 & $195,4 \pm 16,2$ & $200,4 \pm 39,8$ & 0,27 \\
\hline
\end{tabular}

Fuente: elaboración propia

Teniendo en cuenta este valor promedio, la tabla muestra que las BSr y BM1 son las briquetas con la energía de activación mayor, mientras que BEs presenta la energía de activación más baja. Esto indica que la reactividad en la combustión de las BEs es mayor que en el resto de las briquetas, por requerir menor energía para iniciar su incineración. Esto es coherente con los valores de la temperatura pico (Tp) en la zona II, zona que controla la cinética del proceso dado que allí se lleva a cabo la mayor parte de la reacción química, que para la BEs reporta los menores valores de Tp, confirmando la menor energía de activación y la mayor reactividad entre las briquetas en estudio. Respecto al orden de reacción, todas las briquetas, excepto la BSr (orden de reacción $0,33)$, presentan aproximadamente el mismo orden de reacción $(0,27)$.

Los resultados de la regresión lineal de la metodología de Avrami se muestran en la figura 6 , donde se evalúa la dependencia del orden de reacción con la temperatura, 
empleando siete niveles de temperatura a tres velocidades de calentamiento. En la figura se observa que en cada una de las briquetas, el orden de reacción presenta diferentes valores respecto a los niveles de temperatura evaluados. Estos valores, siguen una tendencia similar a medida que aumenta la temperatura de reacción. Dicho comportamiento también es reportados por Gai et al. (2013) [4] y muestra que, aunque los mecanismos de reacción de la combustión no son iguales en las diferentes briquetas, sí tienen un comportamiento similar, sobre todo en un rango de temperatura de 600 a $700{ }^{\circ} \mathrm{C}$.

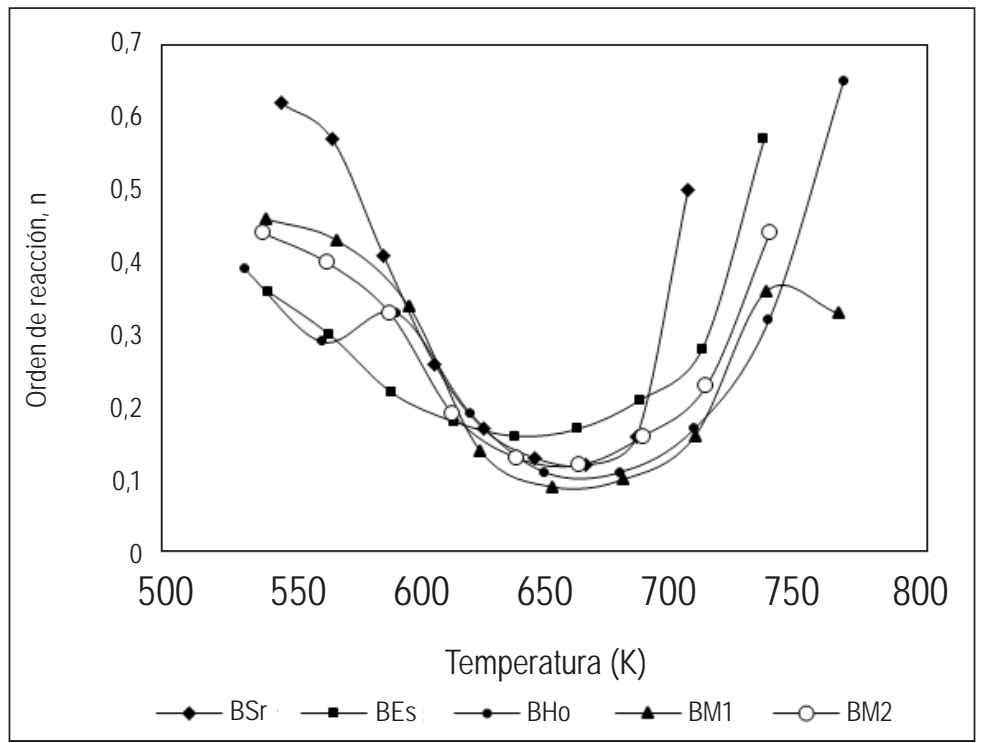

Figura 6. Comportamiento del orden de reacción respecto a la temperatura.

Fuente: elaboración propia

\section{CONCLUSIONES}

El método Starink reporta mayor precisión en los valores calculados de energía de activación respecto a los obtenidos por el método Friedman. Esto se sustenta por los menores valores de la desviación estándar registrados por el método Starink.

Aunque la energía de activación es el parámetro ampliamente reportado en la literatura para determinar la reactividad, esta puede ser complementada con el análisis de las temperaturas de pico como medida de la reactividad.

Se aprecia claramente cómo el índice de combustibilidad (MV/CF) no es suficiente para cuantificar la reactividad porque se debe determinar la energía de activación como parámetro cinético que cuantifica la reactividad. 
Se encontró que de las briquetas preparadas, la BEs es más reactiva a la combustión, debido al menor valor de energía de activación reportado por esta, lo que indica que requiere menor energía para iniciar su incineración. Esto también se comprueba por las menores Tp encontradas en la zona II para esta briqueta.

\section{AGRADECIMIENTOS}

Los autores agradecen a la Universidad Nacional de Colombia por el apoyo económico al proyecto de investigación titulado Evaluación del potencial energético y bioactivo de los residuos generados por la producción y transformación de la uva, con código Hermes 18921, y a la Universidad del Valle por la asistencia de servicios técnicos.

\section{REFERENCIAS}

[1] M. E. Brown, Reaction kinetics from thermal analysis, en Introduction to Thermal Analysis Techniques and Applications, New York: Kluwer Academic Publishers, pp. 181-214, 2004.

[2] J. B. Dahiya, et al., "Kinetics of isothermal and non-isothermal degradation of cellulose: model-based and model-free methods", Polymer International, vol. 57, pp. 722-729, 2008.

[3] M. Valente, et al., "Investigation of grape marc combustion using thermogravimetric analysis. Kinetic modeling using an extended independent parallel reaction (EIPR)", Fuel Processing Technology, vol. 131, pp. 297-303, 2015.

[4] C. Gai, et al., "The kinetic analysis of the pyrolysis of agricultural residue under nonisothermal conditions”, Bioresource Technology, vol. 127, pp. 298-305, 2013.

[5] L. Fiori, et al., "Modeling of the devolatilization kinetics during pyrolysis of grape residues", Bioresource Technology, vol. 103, pp. 389-397, 2012.

[6] A. Anca-Couce, et al., "How to determine consistent biomass pyrolysis kinetics in a parallel reaction scheme", Fuel, vol. 123, pp. 230-240, 2014.

[7] J. E. White, et al., "Biomass pyrolysis kinetics: a comparative critical review with relevant agricultural residue case studies", Journal of Analytical and Applied Pyrolysis, vol. 91, n. ${ }^{\circ}$ 1, pp. 1-33, 2011.

[8] A. C. Deiana, et al., "Use of grape stalk, a waste of the viticulture industry, to obtain activated carbon", Journal of Hazardous Materials, vol. 172, pp. 13-19, 2009.

[9] M. V. Kok and E. Özgür, "Thermal analysis and kinetics of biomass samples", Fuel Processing Technology, vol. 106, pp. 739-743, 2013.

[10] Q. Xu, et al., "A kinetic study on the effects of alkaline earth and alkali metal compounds for catalytic pyrolysis of microalgae using thermogravimetry“, Applied Thermal Engineering, vol. 73 , pp. 355-359, 2014. 
[11] X. Peng, et al., "Thermogravimetric analysis of co-combustion between microalgae and textile dyeing sludge”, Bioresource Technology, vol. 180, pp. 288-295, 2015.

[12] C. Gai, et al., "Combustion behavior and kinetics of low-lipid microalgae via thermogravimetric analysis”, Bioresource Technology, vol. 181, pp. 148-154, 2015.

[13] D. Jelic, et al., "A thermogravimetric study of reduction of silver oxide under non-isothermal conditions", Contemporary Materials, vol. 2, pp. 144-150, 2010.

[14] D. López-González, et al., "Thermogravimetric-mass spectrometric analysis on combustion of lignocellulosic biomass”, Bioresource Technology, vol. 143, pp. 562-574, 2013.

[15] M. Jeguirim and G. Trouvé, "Pyrolysis characteristics and kinetics of Arundo donax using thermogravimetric analysis", Bioresource Technology, vol. 100, pp. 4026-4031, 2009. 